COLLIMATED BIPOLAR OUTFLOW AND THE FORMATION OF NUCLEAR SPIRALS; POSSIBLE ROLE OF MAGNETIC FIELDS

\author{
P. PISMIS, E. MORENO, A. GARCIA-BARRETO \\ Instituto de Astronomia \\ Universidad Nacional Autonoma de Mexico \\ Apdo. Postal 70-264, 04510 Mexico, D.F., Mexico
}

The existence of non-steady phenomena, namely activity in the form of radial motions (outflow) of matter from the nuclei of galaxies is well established at present. Active Galactic Nuclei (AGN) constitute a topic of great interest and are intensively studied by all existing observational techniques. Conventionally objects classified as AGN span a range from quasars, radio galaxies to seyferts 1 and 2 . It appears, however, that there exist galaxies which exhibit somewhat milder activity which does not qualify their inclusion in the AGN group. The designation of MAGN (M for mildly) was suggested in the past (Pismis, 1986) to cover the less energetic nuclei. It may be reasonable to consider that active nuclei form a sequence, the difference along it being due to the energetics of the nuclei, from the most active quasars and radio galaxies down to the mildest ones like M31 or our Galaxy. The phenomenon underlying the activity may thus be universal, subject to the intrinsic energetics of the nuclei (Pismis, 1987).

We argue in this contribution that the very tight bright spiral features in the nuclei of generally barred galaxies provide evidence for past (or ongoing?) nuclear activity.

We mention here that such structures are referred to as nuclear "rings" but high resolution unsaturated images show diametrically opposite details suggesting a tight spiral instead. Incidentally, it is easier for Nature to create spirals (as we shall see later) rather than "rings". We show in what follows that tight spirals can be formed easily by the collimated outflow of plasma from regions diametrically opposite each other on a rotating nucleus of a galaxy.

\title{
The Model
}

The two mechanisms underlying the model are (a) the collimated bisymmetrical outflow of plasma; jets and lobes shown by AGN support this assumption. Further, we assume that the regions of outflow are in the equator of the nucleus; (b) the rotation of a nucleus, a phenomenon so universal as not to need any further comment.

\section{Analytical Derivation of the Tight Spiral}

We assume the nucleus to be a point-like mass. Ejecta in the presence of this central mass describe conic sections. Here we derive the locus of the 447

R. Beck et al. (eds.), Galactic and Intergalactic Magnetic Fields, 447-448.

(c) 1990 IAU. Printed in the Netherlands. 
ejecta while the nucleus rotates.

The symbols used are as follows: Let $\xi=R / R_{0}$, the radius vector, $R_{0}$ radius at ejection, $e=$ eccentricity $\left(V_{\text {e ject }} / V_{\text {rot }}\right), V_{\text {rot }}=\left(G M / R_{0}\right)^{1 / 2}$. Case 1: e $<1$. The ejecta describe elliptical orbits. The equation of the locus is as follows:

$\beta=\arccos \frac{1}{2}\left(a-\frac{!}{\xi}\right)-\left(1-\mathrm{e}^{2}\right)^{-3 / 2}\left\{e\left[|\cos \alpha|-\sqrt{1-\mathrm{e}^{2}}\right]+\alpha-\operatorname{arc} \sin \mathrm{e}\right\}-\frac{\pi}{2}$ $\alpha=\arcsin \frac{1}{2}\left\{1-\xi\left(1-\mathrm{e}^{2}\right)\right\}$

Case 2: e > 1. The ejecta describe hyperbolic orbits. The equation for this case is given in an earlier publication.

\section{Numerical Results}

We have computed the locus for the central mass of $5 \times 10^{9} \mathrm{M}_{\odot}$ and for $\mathrm{e}=0.7$ while the duration of ejection is $\approx 10^{7}$ years. Figure 1 is the double locus inclined by $40^{\circ}$. It resembles closely the central spiral of NGC 4736 given in Figure 2.

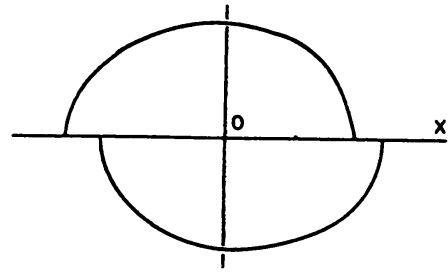

Fig. 1. Locus of the double spiral drawn for case 1 and inclined by $40^{\circ}$ to simulate Fig. 2

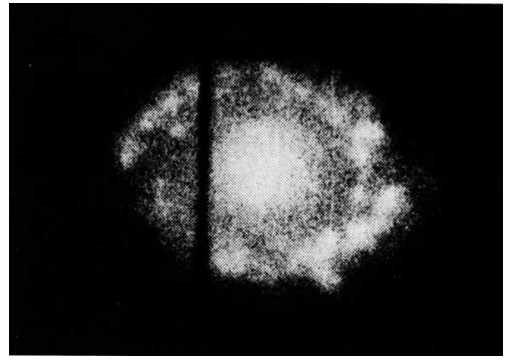

Fig. 2. Central spiral of NGC 4736 taken in $\mathrm{H} \alpha$ with the $2.1 \mathrm{~m}$ reflector at the SPM Observatory, Mexico

We believe that the bi-symmetry of ejection is due to the existence of a dipole-like magnetic field permeating the nucleus; plasma will thus be funneled essentially through the magnetic poles.

Our model reasonably accounts for the following properties of the central spiral:

1) the morphology

2) the existence of outward motions as well as inward motions varying along the spiral pattern.

Magnetic lines of force along the spiral pattern are expected to cause non-thermal radiation; indeed our observations in $6 \mathrm{~cm}$ and $20 \mathrm{~cm}$ continuum with the VLA have shown the mostly non-thermal components which delineate reasonably well the tight spirals essentially of NGC 4314 and NGC 613, among others. Polarization measurements of these small structures have not given conclusive results. Further investigations in this direction should be rewarding. 